\title{
SPECIAL SOLUTIONS TO SOME KOLMOGOROV EQUATIONS ARISING FROM CUBIC SENSOR PROBLEMS*
}

\author{
STEPHEN S.T. YAU ${ }^{\dagger}$, RUXU DU ${ }^{\ddagger}$, AND LIXING JIA
}

1. Introduction. Ever since the technique of the Kalman-Bucy filter was popularized, there has been an intense interest in developing nonlinear filtering theory. Basically we have a signal or state process $x=\left\{x_{t}\right\}$ which is usually not observable. What we can observe is a related process $y=\left\{y_{t}\right\}$. The goal of nonlinear filtering is to determine the conditional expectation of the form $E\left[\phi\left(x_{t}\right): y_{s}, 0 \leq s \leq t\right]$ where $\phi$ is any $C^{\infty}$ function or even better to compute the entire conditional probability density $\rho(t, x)$ of $x_{t}$ given the observation history $\left\{y_{s}: 0 \leq s \leq t\right\}$. In practical applications, it is preferable that the computation of conditional probability density be preformed recursively in terms of a statistic $\theta=\left\{\theta_{t}\right\}$, which can be updated by using only the latest observations.

In some cases, $\theta_{t}$ is computable with a finite system of differential equations driven by $y$. This leads to the ideal notion of finite dimensional recursive filter. By definition such a filter is a system:

$$
d \theta_{t}=\alpha\left(\theta_{t}\right) d t+\sum_{i=1}^{p} \beta_{i}\left(\theta_{t}\right) d y_{i t}
$$

driven by the observation $y_{i t}$ where $y_{i t}$ is the i-th component of $y_{t}, i=1, \cdots, p$; together with an output map

$$
E\left[\phi\left(x_{t}\right): y_{s}, 0 \leq s \leq t\right]=\gamma\left(\theta_{t}\right)
$$

In the 1960s and early 1970s, the basic approach to nonlinear filtering theory was via "innovation methods" originally proposed by Kailath [Ka] and Frost and Kailath [Fr-Ka] and subsequently rigorous developed by Fujisaki, Kallianpur, and Kunita [F-K-K] in 1972. As pointed out by Mitter [Mi], the difficulty with this approach is that innovation process is not, in general, explicitly computable (except

\footnotetext{
*Dedicated to Professor T. E. Duncan on the occasion of sixty five birthday.

$\dagger$ Department of Mathematics, Statistics and Computer Science, University of Illinois at Chicago, 851 S. Morgan Street, Chicago, IL, 60607-7045, USA.

${ }^{\ddagger}$ Department of Automation and Computer-Aided Engineering, The Chinese University of Hong Kong, Shatin, N. T., Hong Kong.

$\S$ Department of Mathematics and Computer Science, Chicago State University, Chicago, IL, 60628.
} 
in the well-known Kalman-Bucy case). In the late 1970s, Brockett and Clark [Br$\mathrm{Cl}$, Brockett [Br] and Mitter [Mi] proposed the idea of using estimation algebras to construct a finite-dimensional nonlinear filter. In 1983 Brockett proposed to classify all finite dimensional estimation algebras. After many years hard work, Yau and his coworkers have finally classified all finite dimensional estimation algebras of maximal rank ([Ya-Hu1], [Ch-Ya], [Y-W-W]). In particular Yau and Hu [Ya-Hu1] proved that the existence of finite dimensional estimation algebra of maximal rank will imply that the drift term of the state dynamic is an affine vector field plus a gradient vector field and the drift term of the observation dynamic is an affine vector field. In [Ya] (see also [Ya-Hu2]) Yau gave sufficient conditions for existence of finite dimensional nonlinear filter.

Despite the success of the classification of finite dimensional estimation algebras of maximal rank, the problem of classification of non-maximal rank finite dimensional estimation algebras is still wide open except for the case of state space dimension 2 which was finished by $\mathrm{Wu}$ and $\mathrm{Yau}[\mathrm{Wu}-\mathrm{Ya}]$ and some construction of non-maximal rank finite dimensional algebras by Rasoulion and Yau [Ra-Ya]. Due to the difficulty of the problem, Brockett suggested that one should understand the low dimensional estimation algebras first. In [Ya-Ra], Yau and Rasoulian have classified estimation algebras of dimension at most four. Recently, Chiou, Chiueh and Yau [C-C-Y] gave a structure theorem for estimation algebras of dimension five. Using this structure theorem, they have found a new class of finite dimensional estimation algebras.

Although it is an interesting and challenging problem to classify all finite dimensional filters, it appears that from Yau's previous woks, finite dimensional filter simply does not exist for many practical situations. In [Ya-Ya1], Yau and Yau proved the existence and decay estimates of the solution to the DMZ (Duncan-Mortensen-Zakai) equation under the assumption that the drift terms $f(x)$ and $h(x)$ of the signal dynamic and observation dynamic respectively have linear growths. Later they [Ya-Ya2] showed that the real-time solution of DMZ equation can be reduced to off-time solution of Kolmogorov equation if $f(x)$ and $h(x)$ have linear growths. However, the results of [Ya-Ya2] do not cover the cubic sensor problem which has received considerable attention in the past (see for examples [Ca-Ge], [Bu-Pa], [H-M-S], [Oc], [S-B-S], and $[\mathrm{Su}]$ ). Recently Yau and Yau [Ya-Ya3] finally proved that the real-time solution of DMZ equation can be reduced to off-time solution of Kolmogorov equation if the growth of the drift term of observation dynamic at infinity is faster than the growth of the drift term of signal dynamic at infinity. Thus they have theoretically solved the nonlinear filtering problems which one encounters in practical environments.

In view of Yau-Yau's theoretical solution to the nonlinear filtering problem, it is natural for us to ask how to solve the Kolmogorov equation associated to the 
signal observation model in nonlinear filtering theory. In this paper we consider the Kolmogorov equation arising from the cubic sensor problem. Let $f(x)=a x+b$ be the drift term of the state dynamic and $h(x)=h_{0} x^{3}+h_{1} x^{2}+h_{2} x+h_{3}$ be the drift term of the observation dynamic. Here $a, b, h_{0}, h_{1}, h_{2}$ and $h_{3}$ are constants. Thus there is a 6 dimensional family of Kolmogorov equations with parameters $a, b, h_{0}, h_{1}, h_{2}$ and $h_{3}$. We shall show that there is a 4 dimensional subfamily of these Kolmogorov equations which admit closed form solution.

In section 2, we shall recall the basic filtering problem. In section 3, we review the reduction from robust DMZ equation to Kolmogorov equation. In section 4, we shall consider the 7 dimensional family of Kolmogorov equations arising from the cubic sensor problem. It is interesting to find solutions of these Kolmogorov equations because the solutions of these Kolmogorov equations will give us the best predictions of the signals without observation data available.

2. Basic Filtering Problem. The filtering problem considered here is based on the following signal observation model in Ito form:

$$
\left\{\begin{aligned}
d x(t) & =f(x(t)) d t+g(x(t)) d v(t) & x(0)=x_{0} \\
d y(t) & =h(x(t)) d t+d w(t) & y(0)=0
\end{aligned}\right.
$$

in which $x, v, y$ and $w$ are, respectively, $R^{n}, R^{p}, R^{m}$ and $R^{m}$ valued processes and $\mathrm{v}$ and $\mathrm{w}$ independent, standard Brownian processes. We further assume that $n=p ; f$, $g$, and $\mathrm{h}$ are vector-valued, orthogonal matrix-valued and vector-valued $C^{\infty}$ smooth functions. We shall refer to $x(t)$ as the state and $y(t)$ as the observation at time $t$.

Let $\rho(t, x)$ denote the conditional probability density of the state given the observation $\{y(s): 0 \leq s \leq t\}$. It is well known that $\rho(t, x)$ is given by normalizing a function $\sigma(t, x)$ that satisfies the following DMZ equation in Fisk-Stratonovich form:

$$
\left\{\begin{array}{l}
d \sigma(t, x)=L_{0} \sigma(t, x) d t+\sum_{i=1}^{m} L_{i} \sigma(t, x) d y_{i}(t) \\
\sigma(0, x)=\sigma_{0}(x)
\end{array}\right.
$$

where

$$
L_{0}=\frac{1}{2} \sum_{i=1}^{n} \frac{\partial^{2}}{\partial x_{i}^{2}}-\sum_{i=1}^{n} f_{i}(x) \frac{\partial}{\partial x_{i}}-\sum_{i=1}^{n} \frac{\partial f_{i}}{\partial x_{i}}(x)-\frac{1}{2} \sum_{i=1}^{m} h_{i}^{2}(x),
$$

and for $i=1, \ldots, m, L_{i}$ is the zero-degree differential operator of multiplication by $h_{i}$ and $\sigma_{0}$ is the probability density of the initial point $x_{0}$.

Davis introduced a new unnormalized density

$$
u(t, x)=\exp \left(-\sum_{i=1}^{m} h_{i}(x) y_{i}(t)\right) \sigma(t, x)
$$


He reduced (2.2) to the following time-varying partial differential equation which is called robust DMZ-equation

$$
\left\{\begin{aligned}
& \frac{\partial u}{\partial t}(t, x)= L_{0} u(t, x)+\sum_{i=1}^{m} y_{i}(t)\left[L_{0}, L_{i}\right] u(t, x) \\
& \quad+\frac{1}{2} \sum_{i, j=1}^{m} y_{i}(t) y_{j}(t)\left[\left[L_{0}, L_{i}\right], L_{j}\right] u(t, x) \\
& u(0, x)=\sigma_{0}(x)
\end{aligned}\right.
$$

where $[\mathrm{A}, \mathrm{B}]$ is the Lie bracket of the operators A and B. It is easy to show that (2.4) is equivalent to the following time-varying partial differential equation:

$$
\left\{\begin{aligned}
\frac{\partial u}{\partial t}(t, x)= & \frac{1}{2} \sum_{i=1}^{n} \frac{\partial^{2} u}{\partial x_{i}^{2}}(t, x)+\sum_{i=1}^{n}\left(-f_{i}(x)+\sum_{j=1}^{m} y_{j}(t) \frac{\partial h_{j}}{\partial x_{i}}(x)\right) \frac{\partial u}{\partial x_{i}}(t, x) \\
& -\left[\sum_{i=1}^{n} \frac{\partial f_{i}}{\partial x_{i}}(x)+\frac{1}{2} \sum_{i=1}^{m} h_{i}^{2}(x)-\frac{1}{2} \sum_{i=1}^{m} y_{i}(t) \Delta h_{i}(x)\right. \\
& \left.+\sum_{i=1}^{m} \sum_{j=1}^{n} y_{i}(t) f_{i}(x) \frac{\partial h_{j}}{\partial x_{i}}(x)-\frac{1}{2} \sum_{i, j=1}^{m} \sum_{k=1}^{n} y_{i}(t) y_{j}(t) \frac{\partial h_{i}}{\partial x_{k}}(x) \frac{\partial h_{j}}{\partial x_{k}}(x)\right] u(t, x) \\
u(0, x)= & \sigma_{0}(x) .
\end{aligned}\right.
$$

3. Reduction from robust DMZ equation to Kolmogorov equation. The fundamental problem of nonlinear filtering theory is how to solving the robust DMZ equation (2.5) in real time and in memoryless manner. In this section, we shall describe our algorithm which achieves this goal for a large class of filtering system with arbitrary initial distribution by reducing it to solve Kolmogorov equation. Our algorithm is based on the following observation.

Proposition 3.1. For any $\tau_{1}, \tau_{2}$ with $\tau_{1}<\tau_{2}, u(t, x)$ satisfies the following Kolmogorov equation

$$
\frac{\partial \tilde{u}}{\partial t}(t, x)=\frac{1}{2} \Delta \tilde{u}(t, x)-\sum_{i=1}^{n} f_{i}(x) \frac{\partial \tilde{u}}{\partial x_{i}}(t, x)-\left(\sum_{i=1}^{n} \frac{\partial f_{i}}{\partial x_{i}}(x)+\frac{1}{2} \sum_{i=1}^{m} h_{i}^{2}(x)\right) \tilde{u}(t, x)
$$

For $\tau_{1} \leq t \leq \tau_{2}$ if and only if

$$
u(t, x)=e^{-\sum_{i=1}^{m} y_{i}\left(\tau_{2}\right) h_{i}(x)} \tilde{u}(t, x)
$$


satisfies the robust DMZ equation with observation being fixed at $y\left(\tau_{1}\right)$

$$
\begin{aligned}
& \frac{\partial u}{\partial t}(t, x)=\frac{1}{2} \Delta u(t, x)+\sum_{i=1}^{n}\left(-f_{i}(x)+\sum_{j=1}^{m} y_{j}\left(\tau_{2}\right) \frac{\partial h_{j}}{\partial x_{i}}(x)\right) \frac{\partial u}{\partial x_{i}}(t, x) \\
& -\left(\sum_{i=1}^{n} \frac{\partial f_{i}}{\partial x_{i}}(x)+\frac{1}{2} \sum_{i=1}^{m} h_{i}^{2}(x)-\frac{1}{2} \sum_{i=1}^{m} y_{i}\left(\tau_{2}\right) \Delta h_{i}(x)+\sum_{i=1}^{m} \sum_{j=1}^{n} y_{i}\left(\tau_{2}\right) f_{j}(x) \frac{\partial h_{i}}{\partial x_{j}}(x)\right. \\
& \left.-\frac{1}{2} \sum_{k=1}^{n} \sum_{i, j=1}^{m} y_{i}\left(\tau_{2}\right) y_{j}\left(\tau_{2}\right) \frac{\partial h_{i}}{\partial x_{k}}(x) \frac{\partial h_{j}}{\partial x_{k}}(x)\right) u(t, x)
\end{aligned}
$$

Proof. It is straight forward to show that

$$
\begin{aligned}
& e^{\sum_{i=1}^{m} y_{i}\left(\tau_{1}\right) h_{i}(x)}\left[-\frac{\partial}{\partial t}+\frac{1}{2} \Delta+\sum_{i=1}^{n}\left(-f_{i}(x)+\sum_{j=1}^{m} y_{j}\left(\tau_{2}\right) \frac{\partial h_{j}}{\partial x_{i}}\right) \frac{\partial}{\partial x_{i}}\right. \\
& -\left(\sum_{i=1}^{n} \frac{\partial h_{i}}{\partial x_{i}}(x)+\frac{1}{2} \sum_{i=1}^{m} h_{i}^{2}(x)-\frac{1}{2} \sum_{i=1}^{m} y_{i}\left(\tau_{2}\right) \Delta h_{i}(x)\right. \\
& +\sum_{i=1}^{m} \sum_{j=1}^{n} y_{i}\left(\tau_{2}\right) f_{j}(x) \frac{\partial h_{i}}{\partial x_{j}}(x) \\
& \left.\left.-\frac{1}{2} \sum_{k=1}^{n} \sum_{i, j=1}^{m} y_{i}\left(\tau_{2}\right) y_{j}\left(\tau_{2}\right) \frac{\partial h_{i}}{\partial x_{k}} \frac{\partial h_{i}}{\partial x_{k}}\right)\right] u(t, x) \\
= & -\frac{\partial \tilde{u}}{\partial t}(t, x)+\frac{1}{2} \Delta \tilde{u}(t, x)-\sum_{i=1}^{n} f_{i}(x) \frac{\partial \tilde{u}}{\partial x_{i}}(t, x) \\
& -\left(\sum_{i=1}^{n} \frac{\partial f_{i}}{\partial x_{i}}(x)+\frac{1}{2} \sum_{i=1}^{m} h_{i}^{2}(x)\right) \tilde{u}(t, x)
\end{aligned}
$$

Proposition (3.1) follows immediately from (3.3).

We remark that (3.2) is obtained from robust DMZ equation by freezing the observation $y(t)$ to $y\left(\tau_{1}\right)$. Basing on Proposition (3.1), we shall formulate our algorithm to solve robust DMZ equation and in the appendix of [Ya-Ya3] it is shown that the solution of out algorithm approximate the solution of robust DMZ equation very well in $L^{1}$ sense.

Suppose that $u(t, x)$ is the solution of robust DMZ equation and we want to compute $u(\tau, x)$. Let $P_{k}=\left\{0=\tau_{0}<\tau_{1}<\tau_{2}<\ldots<\tau_{k}=\tau\right\}$ be a partition of $[0, \tau]$. Let $u_{i}(t, x)$ be a solution of the following partial differential equation for $\tau_{i-1} \leq t \leq \tau_{i}$. 


$$
\left\{\begin{aligned}
\frac{\partial u_{i}}{\partial t}( & t, x)=\frac{1}{2} \Delta u_{i}(t, x)+\sum_{l=1}^{n}\left(-f_{l}(x)+\sum_{j=1}^{m} y_{j}\left(\tau_{i}\right) \frac{\partial h_{j}}{\partial x_{l}}(x)\right) \frac{\partial u_{i}}{\partial x_{l}}(t, x) \\
- & \left(\sum_{l=1}^{n} \frac{\partial f_{l}}{\partial x_{l}}(x)+\frac{1}{2} \sum_{l=1}^{m} h_{l}^{2}(x)-\frac{1}{2} \sum_{j=1}^{m} y_{j}\left(\tau_{i}\right) \Delta h_{j}(x)\right. \\
+ & \sum_{j=1}^{m} \sum_{l=1}^{n} y_{j}\left(\tau_{i}\right) f_{l}(x) \frac{\partial h_{j}}{\partial x_{l}}(x) \\
- & \left.\frac{1}{2} \sum_{p=1}^{n} \sum_{j, l=1}^{m} y_{j}\left(\tau_{i}\right) y_{l}\left(\tau_{i}\right) \frac{\partial h_{j}}{\partial x_{p}}(x) \frac{\partial h_{l}}{\partial x_{p}}(x)\right) u_{i}(t, x) \\
u_{i}\left(\tau_{i-1}\right) & =u_{i-1}\left(\tau_{i-1}, x\right) .
\end{aligned}\right.
$$

Define the norm of the partition $P_{k}$ by $\left|P_{k}\right|=\sup _{1 \leq i \leq k}\left\{\left|\tau_{i}-\tau_{i-1}\right|\right\}$. In [Ya-Ya2], we proved that in $L^{1}$ sense

$$
u(\tau, x)=\lim _{\left|P_{k}\right| \rightarrow 0} u_{k}(\tau, x)
$$

Therefore it remains to describe an algorithm to compute $u_{k}\left(\tau_{k}, x\right)$. By Proposition $3.1, u_{1}\left(\tau_{1}, x\right)$ can be computed by $u_{1}\left(\tau_{1}, x\right)$ where $u_{1}(t, x)$ for $0 \leq t \leq \tau_{1}$ satisfies the following Kolmogorov equation

$$
\left\{\begin{aligned}
\frac{\partial \tilde{u}_{1}}{\partial t}(t, x)= & \frac{1}{2} \Delta \tilde{u}_{1}(t, x)-\sum_{j=1}^{n} f_{i}(x) \frac{\partial \tilde{u}_{1}}{\partial x_{i}}(x) \\
& -\left(\sum_{j=1}^{n} \frac{\partial f_{i}}{\partial x_{i}}(x)+\frac{1}{2} \sum_{j=1}^{m} h_{j}^{2}(x)\right) \tilde{u}_{1}(t, x) \\
\tilde{u}_{1}(0, x)= & \sigma_{0}(x) e^{\sum_{j=1}^{m} y_{j}\left(\tau_{1}\right) h_{j}(x)} .
\end{aligned}\right.
$$

In fact, by the uniqueness of the solution of Kolmogorov equation, we have

$$
u_{1}(t, x)=\tilde{u}_{1}(t, x), \quad 0 \leq t \leq \tau_{1}
$$

In general, Proposition 3.1 tells us that for $i \geq 2, u_{i}\left(\tau_{i}, x\right)$ can be computed by $u_{i}\left(\tau_{i}, x\right)$ where $u_{i}(t, x)$ for $\tau_{i-1} \leq t \leq \tau_{i}$ satisfies the following Kolmogorov equation $(3.8)$

$$
\left\{\begin{array}{l}
\frac{\partial \tilde{u}_{i}}{\partial t}(t, x)=\frac{1}{2} \Delta \tilde{u}_{i}(t, x)-\sum_{j=1}^{n} f_{j}(x) \frac{\partial \tilde{u}_{i}}{\partial x_{j}}(t, x)-\left(\sum_{j=1}^{n} \frac{\partial f_{j}}{\partial x_{j}}(x)+\frac{1}{2} \sum_{j=1}^{m} h_{j}^{2}(x)\right) \tilde{u}_{i}(t, x) \\
\tilde{u}_{i}\left(\tau_{i-1}, x\right)=e^{\sum_{j=1}^{m}\left(y_{j}\left(\tau_{i}\right)-y_{j}\left(\tau_{i-1}\right)\right) h_{j}(x)} \tilde{u}_{i-1}\left(\tau_{i-1}, x\right)
\end{array}\right.
$$

where the last initial condition comes from

$$
\begin{aligned}
\tilde{u}_{i}\left(\tau_{i-1}, x\right) & =u_{i}\left(\tau_{i-1}, x\right) e^{\sum_{j=1}^{m} y_{j}\left(\tau_{i}\right) h_{j}(x)}=u_{i-1}\left(\tau_{i-1}, x\right) e^{\sum_{j=1}^{m} y_{j}\left(\tau_{i}\right) h_{j}(x)} \\
& =e^{\sum_{j=1}^{m}\left(y_{j}\left(\tau_{i}\right)-y_{j}\left(\tau_{i-1}\right)\right) h_{j}(x)} \tilde{u}_{i-1}\left(\tau_{i-1}, x\right) .
\end{aligned}
$$


In fact, we have

$$
u_{i}\left(\tau_{i}, x\right)=e^{-\sum_{j=1}^{m} y_{j}\left(\tau_{i}\right) h_{j}(x)} \tilde{u}_{i}\left(\tau_{i}, x\right) .
$$

In the view of (2.3), (3.5) and (3.9), we have the following theorem.

Theorem 3.2. The unnormalized density $\sigma$ can be computed via solution u of Kolmogorov equation (3.8). More specifically,

$$
\sigma(\tau, x)=\lim _{\left|P_{k}\right| \rightarrow 0} \tilde{u}_{k}\left(\tau_{k}, x\right)
$$

Proof.

$$
\begin{aligned}
\sigma(\tau, x)= & u(\tau, x) \exp \left(\sum_{i=1}^{m} h_{i}(x) y_{i}(\tau)\right) \\
& =\lim _{\left|P_{k}\right| \rightarrow 0} u_{k}(\tau, x) \exp \left(\sum_{i=1}^{m} h_{i}(x) y_{i}(\tau)\right),
\end{aligned}
$$

where $P_{k}=\left\{0=\tau_{0}<\tau_{1}<\ldots<\tau_{k}=\tau\right\}$.

In view of (3.9), we have

$$
\begin{aligned}
\sigma(\tau, x)= & \lim _{\left|P_{k}\right| \rightarrow 0} e^{-\sum_{i=1}^{m} y_{j}\left(\tau_{k}\right) h_{j}(x)} \tilde{u}_{k}(\tau, x) e^{\sum_{i=1}^{m} h_{i}(x) y_{i}(\tau)} \\
& =\lim _{\left|P_{k}\right| \rightarrow 0} \tilde{u}_{k}(\tau, x)
\end{aligned}
$$

Observe that in our algorithm at step $i$, we only need the observation at time $\tau_{i}$ and $\tau_{i-1}$. We do not need any other previous observation data. Observe also that the Kolmogorov equation (3.8) is uniform for all time steps and it depends on observation $y(t)$ only via initial condition.

\section{Finite dimensional computation of certain Kolmogorov equations.}

In this section, we shall consider the filtering system (2.1) with $n=1$ and affine drift

$$
f(x)=a x+b
$$

and nonlinear observation

$$
h(x)=h_{0} x^{3}+h_{1} x^{2}+h_{2} x+h_{3}
$$

where $a, b, h_{0}, h_{1}, h_{2}$, and $h_{3}$ are constants. This is the famous cubic sensor problem and it is well known that there is no finite dimensional filter for this problem. The Kolmogorov equation associated to this cubic sensor problem is of the following form:

$$
\left\{\begin{aligned}
\frac{\partial u}{\partial x}(t, x)= & \frac{1}{2} \frac{\partial^{2} u}{\partial x^{2}}(t, x)-f(x) \frac{\partial u}{\partial x}(t, x)-\left(\frac{\partial f}{\partial x}(x)+\frac{1}{2} h^{2}(x)\right) u(t, x) \\
= & \frac{1}{2} \frac{\partial^{2} u}{\partial x^{2}}(t, x)-(a x+b) \frac{\partial u}{\partial x}(t, x) \\
& -\left[a+\frac{1}{2}\left(h_{0} x^{3}+h_{1} x^{2}+h_{2} x+h_{3}\right)^{2}\right] u(x, t) \\
u(0, x)= & \sigma_{0}(x)
\end{aligned}\right.
$$


The solution of (4.3) will give us the best predict of the signal without any observation. Notice that equation (4.3) consists of 6 parameters $a, b, h_{0}, h_{1}, h_{2}$, and $h_{3}$. We shall show that there is a 4-dimensional subfamily of equation (4.3) which admits closed form solution.

THEOREM 4.1. Consider the Kolmogorov equation (4.3) arising from cubic sensor problem. Suppose $h_{0}>0$ and $a, b, h_{0}, h_{1}, h_{2}$, and $h_{3}$ satisfy the following two algebraic equations:

$$
\begin{gathered}
\frac{1}{2}\left(a-h_{2}\right)^{2}-\frac{h_{1}}{h_{0}}\left(h_{1} h_{2}+b h_{0}-h_{0} h_{3}-h_{1} h_{2}\right)-\frac{3}{2} h_{0}-a\left(a-h_{2}\right)+b h_{1}-\frac{1}{2} h_{2}^{2}-h_{1} h_{2}=0 \\
\frac{h_{2}}{h_{0}}\left(h_{1} h_{2}+b h_{0}-h_{0} h_{3}-h_{1} h_{2}\right)-h_{1}-b\left(a-h_{2}\right)-h_{2} h_{3}=0
\end{gathered}
$$

Then (4.3) admits the following finite dimensional solution

$$
u(t, x)=e^{c_{0} x^{4}+c_{1} x^{3}+c_{2} x^{2}+c_{3} x+c_{4}}
$$

where

$$
\begin{aligned}
& c_{0}=-\frac{1}{4} h_{0} \\
& c_{1}=-\frac{1}{3} h_{1} \\
& c_{2}=\frac{1}{2}\left(a-h_{2}\right) \\
& c_{3}=\frac{1}{h_{0}}\left(h_{1} h_{2}+b h_{0}-h_{0} h_{3}-h_{1} h_{2}\right) \\
& c_{4}=\quad \frac{1}{2 h_{0}^{2}}\left(h_{1} h_{2}+b h_{0}-h_{0} h_{3}-h_{1} h_{2}\right)^{2}+\frac{1}{2}\left(a-h_{2}\right) \\
&\left.-\frac{b}{h_{0}}\left(h_{1} h_{2}+b h_{0}-h_{0} h_{3}-h_{1} h_{2}\right)-\frac{1}{2} h_{3}^{2}-a\right] t+c_{4}(0)
\end{aligned}
$$

Proof. We first differentiate (4.6) with respect to $t$. We have

$$
\frac{\partial u}{\partial t}(t, x)=\left(\frac{d c_{0}}{d t} x^{4}+\frac{d c_{1}}{d t} x^{3}+\frac{d c_{2}}{d t} x^{2}+\frac{d c_{3}}{d t} x+\frac{d c_{4}}{d t}\right) e^{c_{0} x^{4}+c_{1} x^{3}+c_{2} x^{2}+c_{3} x+c_{4}}
$$

We next differentiate (4.6) with respect to $x$ twice. We have

$$
\begin{aligned}
\frac{\partial u}{\partial x}(t, x)= & \left(4 c_{0} x^{3}+3 c_{1} x^{2}+2 c_{2} x+c_{3}\right) e^{c_{0} x^{4}+c_{1} x^{3}+c_{2} x^{2}+c_{3} x+c_{4}} \\
\frac{\partial^{2} u}{\partial x^{2}}(t, x)= & {\left[\left(4 c_{0} x^{3}+3 c_{1} x^{2}+2 c_{2} x+c_{3}\right)^{2}+12 c_{0} x^{2}+6 c_{1} x\right.} \\
& \left.+2 c_{2}\right] e^{c_{0} x^{4}+c_{1} x^{3}+c_{2} x^{2}+c_{3} x+c_{4}} \\
= & {\left[16 c_{0}^{2} x^{6}+24 c_{0} c_{1} x^{5}+\left(9 c_{1}^{2}+16 c_{0} c_{2}\right) x^{4}+\left(8 c_{0} c_{3}+12 c_{1} c_{2}\right) x^{3}\right.} \\
& +\left(4 c_{2}^{2}+6 c_{1} c_{3}+12 c_{0}\right) x^{2}+\left(4 c_{2} c_{3}+6 c_{1}\right) x \\
& \left.+c_{3}^{2}+2 c_{2}\right] e^{c_{0} x^{4}+c_{1} x^{3}+c_{2} x^{2}+c_{3} x+c_{4}}
\end{aligned}
$$




$$
\begin{aligned}
f(x) \frac{\partial \tilde{u}}{\partial x}= & (a x+b)\left(4 c_{0} x^{3}+3 c_{1} x^{2}+2 c_{2} x+c_{3}\right) e^{c_{0} x^{4}+c_{1} x^{3}+c_{2} x^{2}+c_{3} x+c_{4}} \\
= & {\left[4 a c_{0} x^{4}+\left(3 a c_{1}+4 b c_{0}\right) x^{3}+\left(2 a c_{2}+3 b c_{1}\right) x^{2}\right.} \\
& \left.+\left(a c_{3}+2 b c_{2}\right) x+b c_{3}\right] e^{c_{0} x^{4}+c_{1} x^{3}+c_{2} x^{2}+c_{3} x+c_{4}}
\end{aligned}
$$

$$
\begin{aligned}
\left(\frac{\partial f}{\partial x}+\frac{1}{2} h^{2}(x)\right) u(t, x)= & {\left[a+\frac{1}{2}\left(h_{0} x^{3}+h_{1} x^{2}+h_{2} x+h_{3}\right)^{2}\right] e^{c_{0} x^{4}+c_{1} x^{3}+c_{2} x^{2}+c_{3} x+c_{4}} } \\
= & {\left[\frac{1}{2} h_{0}^{2} x^{6}+h_{0} h_{1} x^{5}+\left(\frac{1}{2} h_{1}^{2}+h_{0} h_{2}\right) x^{4}+\left(h_{0} h_{3}+h_{1} h_{2}\right) x^{3}\right.} \\
& +\left(\frac{1}{2} h_{2}^{2}+h_{1} h_{3}\right) x^{2}+h_{1} h_{3} x+\frac{1}{2} h_{3}^{2} \\
& +a] e^{c_{0} x^{4}+c_{1} x^{3}+c_{2} x^{2}+c_{3} x+c_{4}}
\end{aligned}
$$

From (4.13), (4.14) and (4.15), we have

$$
\begin{aligned}
& \frac{1}{2} \frac{\partial^{2} u}{\partial x^{2}}-f(x) \frac{\partial u}{\partial x}-\left(\frac{\partial f}{\partial x}(x)+\frac{1}{2} h^{2}(x)\right) \tilde{u} \\
& =\left[\left(8 c_{0}^{2}-\frac{1}{2} h_{0}\right) x^{6}+\left(12 c_{0} c_{1}-h_{0} h_{1}\right) x^{5}+\left(\frac{9}{2} c_{1}^{2}+8 c_{0} c_{2}-4 a c_{0}-\frac{1}{2} h_{1}^{2}-h_{0} h_{2}\right) x^{4}\right. \\
& +\left(4 c_{0} c_{3}+6 c_{1} c_{2}-3 a c_{1}-4 b c_{0}-h_{0} h_{3}-h_{1} h_{2}\right) x^{3}+\left(2 c_{2}^{2}+3 c_{1} c_{3}+6 c_{0}-2 a c_{2}\right. \\
& \left.-3 b c_{1}-\frac{1}{2} h_{2}^{2}-h_{1} h_{3}\right) x^{2}+\left(2 c_{2} c_{3}+3 c_{1}-a c_{3}-2 b c_{2}-h_{2} h_{3}\right) x \\
& \left.\left.+\left(\frac{1}{2} c_{3}^{2}+c_{2}-b c_{3}-\frac{1}{2} h_{3}^{2}-a\right)\right)\right] e^{c_{0} x^{4}+c_{1} x^{3}+c_{2} x^{2}+c_{3} x+c_{4}}
\end{aligned}
$$

Comparing (4.12) and (4.16), we obtain the following equations

$$
\begin{aligned}
& 8 c_{0}^{2}-\frac{1}{2} h_{0}^{2}=0 \\
& 12 c_{0} c_{1}-h_{0} h_{1}=0 \\
& \frac{d c_{0}}{d t}=\frac{9}{2} c_{1}^{2}+8 c_{0} c_{2}-4 a c_{0}-\frac{1}{2} h_{1}^{2}-h_{0} h_{2} \\
& \frac{d c_{1}}{d t}=4 c_{0} c_{3}+6 c_{1} c_{2}-3 a c_{1}-4 b c_{0}-h_{0} h_{3}-h_{1} h_{2} \\
& \frac{d c_{2}}{d t}=2 c_{2}^{2}+3 c_{1} c_{3}+6 c_{0}-2 a c_{2}-3 b c_{1}-\frac{1}{2} h_{2}^{2}-h_{1} h_{2} \\
& \frac{d c_{3}}{d t}=2 c_{2} c_{3}+3 c_{1}-a c_{3}-2 b c_{2}-h_{2} h_{3} \\
& \frac{d c_{4}}{d t}=\frac{1}{2} c_{3}^{2}+c_{2}-b c_{3}-\frac{1}{2} h_{3}^{2}-a \\
& (4.17) \Rightarrow \quad c_{0}=-\frac{1}{4} h_{0} \\
& (4.18) \text { and }(4.24) \Rightarrow \quad c_{1}=-\frac{1}{3} h_{1} \\
& (4.19),(4.24) \text { and }(4.25) \Rightarrow \\
& 0=\frac{9}{2}\left(-\frac{1}{3} h_{1}\right)^{2}+8\left(-\frac{1}{4} h_{0}\right) c_{2}-4 a\left(-\frac{1}{4} h_{0}\right)-\frac{1}{2} h_{1}^{2}-h_{0} h_{2} \\
& \Rightarrow c_{2}=\frac{1}{2}\left(a-h_{2}\right)
\end{aligned}
$$

(4.20), (4.26), (4.25) and (4.24) $\Rightarrow$

$$
\begin{aligned}
0= & 4\left(-\frac{1}{4} h_{0}\right) c_{3}+6\left(-\frac{1}{3} h_{1}\right)\left[\frac{1}{2}\left(a-h_{2}\right)\right] \\
& -3 a\left(-\frac{1}{3} h_{1}\right)-4 b\left(-\frac{1}{4} h_{0}\right)-h_{0} h_{3}-h_{1} h_{2} \\
\Rightarrow \quad c_{3}= & \frac{1}{h_{0}}\left(h_{1} h_{2}+b h_{0}-h_{0} h_{3}-h_{1} h_{2}\right)
\end{aligned}
$$


(4.21), (4.27), (4.26), (4.25) and (4.24) $\Rightarrow$

$$
\begin{aligned}
0= & 2\left[\frac{1}{2}\left(a-h_{2}\right)\right]^{2}+3\left(-\frac{1}{3} h_{1}\right)\left[\frac{1}{h_{0}}\left(h_{1} h_{2}+b h_{0}-h_{0} h_{3}-h_{1} h_{2}\right)\right] \\
& +6\left(-\frac{1}{4} h_{0}\right)-2 a\left[\frac{1}{2}\left(a-h_{2}\right)\right]-3 b\left(-\frac{1}{3} h_{1}\right)-\frac{1}{2} h_{2}^{2}-h_{1} h_{2} \\
= & \frac{1}{2}\left(a-h_{2}\right)^{2}-\frac{h_{1}}{h_{0}}\left(h_{1} h_{2}+b h_{0}-h_{0} h_{3}-h_{1} h_{2}\right) \\
& -\frac{3}{2} h_{0}-a\left(a-h_{2}\right)+b h_{1}-\frac{1}{2} h_{2}^{2}-h_{1} h_{2}
\end{aligned}
$$

(4.22), (4.27), (4.26), (4.25) and (4.24) $\Rightarrow$

$$
\begin{aligned}
0= & 2\left[\frac{1}{2}\left(a-h_{2}\right)\right]\left[\frac{1}{h_{0}}\left(h_{1} h_{2}+b h_{0}-h_{0} h_{3}-h_{1} h_{2}\right)\right]+3\left[-\frac{1}{3} h_{1}\right] \\
& -a\left[\frac{1}{h_{0}}\left(h_{1} h_{2}+b h_{0}-h_{0} h_{3}-h_{1} h_{2}\right)\right]-2 b\left[\frac{1}{2}\left(a-h_{2}\right)\right]-h_{2} h_{3} \\
= & -\frac{h_{1}}{h_{0}}\left(h_{1} h_{2}+b h_{0}-h_{0} h_{3}-h_{1} h_{2}\right)-h_{1}-b\left(a-h_{2}\right)-h_{2} h_{3}
\end{aligned}
$$

(4.23), (4.24), (4.25), (4.26) and (4.27) $\Rightarrow$

$$
\begin{aligned}
\frac{d c_{4}}{d t}= & \frac{1}{2}\left[\frac{1}{h_{0}}\left(h_{1} h_{2}+b h_{0}-h_{0} h_{3}-h_{1} h_{2}\right)\right]^{2}+\frac{1}{2}\left(a-h_{2}\right) \\
& -b\left[\frac{1}{h_{0}}\left(h_{1} h_{2}+b h_{0}-h_{0} h_{3}-h_{1} h_{2}\right)\right]-\frac{1}{2} h_{3}^{2}-a \\
\Rightarrow \quad c_{4}= & {\left[\frac{1}{2 h_{0}^{2}}\left(h_{1} h_{2}+b h_{0}-h_{0} h_{3}-h_{1} h_{2}\right)^{2}+\frac{1}{2}\left(a-h_{2}\right)\right.} \\
& \left.-\frac{b}{h_{0}}\left(h_{1} h_{2}+b h_{0}-h_{0} h_{3}-h_{1} h_{2}\right)-\frac{1}{2} h_{3}^{2}-a\right] t+c_{4}(0)
\end{aligned}
$$

TheOREm 4.2. Consider the Kolmogorov equation (4.3) arising from cubic sensor problem. Suppose $h_{0}<0$ and $a, b, h_{0}, h_{1}, h_{2}$, and $h_{3}$ satisfy the following two algebraic equations:

(4.30)

$\frac{1}{2}\left(a-h_{2}\right)^{2}+\frac{h_{1}}{h_{0}}\left(-h_{1} h_{2}+b h_{0}+h_{0} h_{3}+h_{1} h_{2}\right)+\frac{3}{2} h_{0}-a\left(a+h_{2}\right)-b h_{1}-\frac{1}{2} h_{2}^{2}-h_{1} h_{2}=0$

$$
\frac{h_{2}}{h_{0}}\left(-h_{1} h_{2}+b h_{0}+h_{0} h_{3}+h_{1} h_{2}\right)+h_{1}-b\left(a+h_{2}\right)-h_{2} h_{3}=0
$$

Then (4.3) admits the following finite dimensional solution

$$
u(t, x)=e^{c_{0} x^{4}+c_{1} x^{3}+c_{2} x^{2}+c_{3} x+c_{4}}
$$

where

$$
\begin{aligned}
c_{0}= & \frac{1}{4} h_{0} \\
c_{1}= & \frac{1}{3} h_{1} \\
c_{2}= & \frac{1}{2}\left(a+h_{2}\right) \\
c_{3}= & \frac{1}{h_{0}}\left(-h_{1} h_{2}+b h_{0}+h_{0} h_{3}+h_{1} h_{2}\right) \\
c_{4}=\quad\left[\frac{1}{2 h_{0}^{2}}\left(-h_{1} h_{2}+b h_{0}+h_{0} h_{3}+h_{1} h_{2}\right)^{2}+\frac{1}{2}\left(a+h_{2}\right)\right. & \left.\quad-\frac{b}{h_{0}}\left(-h_{1} h_{2}+b h_{0}+h_{0} h_{3}+h_{1} h_{2}\right)-\frac{1}{2} h_{3}^{2}-a\right] t+c_{4}(0)
\end{aligned}
$$

Proof. The proof is similar to those given to Theorem 4.1. 


\section{REFERENCES}

[Br] R. W. Brockett, Nonlinear systems and nonlinear estimation theory, in: The Mathematics of Filtering and Identification and Applications, M. Hazewinkel and J.C. Williams (Eds.), Reidel, Dordrecht, (1981), 479-504.

[Br-Cl] R. W. Brockett and J. M. C. Clark, The geometry of the conditional density functions, in: Analysis and Optimization of Stochastic Systems, O. L. R. Jacobs et al (Eds.), Academic Press, New York, (1980) 299-309.

[Bu-Pa] R. S. Bucy And J. PAGes, A priori error bounds for the cubic sensor problem, IEEE Transactions on Automatic Control, AC-23:1(1978), pp. 88-91.

[C-C-Y] W.-L. Chiou, W.-R. Chiuen, And S. S.-T. Yau, Structure theorem for five dimensional estimation algebras, Systems \& Control Lett., 55(2006), pp. 275-281.

[Ca-Ge] F. Carravetta And A. Germani, Suboptimal solutions for the cubic sensor problem, Proceeding of $36^{t h}$ IEEE Conf. Decision and Control, San Diego, CA, Dec., (1997), 4460-4461.

[Ch-Ya] J. Chen And S. S.-T. YAU, Finite dimensional filters with nonlinear drift VI: Linear structure of $\Omega$, Mathematics of Control, Signals and Systems, 9(1996), pp. 370-385.

[F-K-K] M. Fujisaki, G. Kallianpur, And H. Kunita, Stochastic differential equations for the nonlinear filtering problem, Osaka Journal of Mathematics, 2(1972), pp. 19-40.

[Fr-Ka] P. A. Frost And T. Kailath, An innovations approach to least-squares estimation II, IEEE Transactions on Automatic Control, 16(1971), pp. 217-226.

[H-M-S] M. Hazewinkel, S. I. Marcus, and H. J. Sussmann, Nonexistence of finite dimensional filters for conditional statistics of the cubic sensor problem, Systems \& Control Lett., 3:6(1983), pp. 331-340.

[Ka] T. Kailath, An innovations approach to least-squares estimation, Part I: Linear filtering in additive white noise, IEEE Transactions on Automatic Control, 13(1986), pp. 646-655.

[Mi] S. K. MitTAR, On the analogy between mathematical problems of nonlinear filtering and quantum physics, Ricerche Automat., 10(1979), pp. 163-216.

[Oc] D. Ocone, Probability densities for conditional statistics in the cubic sensor problem, Mathematics Control Signals Systems, 1(1988), pp. 183-202.

[Ra-Ya] A. Rasoulian And S. S.-T. Yau, Finite dimensional filters with nonlinear drift IX: Construction of finite dimensional estimation algebra of non-maximal rank, Systems $\&$ Control Lett., 30, (1997), 109-118.

[S-B-S] Y. Steiberg, Z. Bobrowsky, And Z. Schuss, On the optimal filtering problem for the cubic sensor, Circuits Systems Signal Process, 7:3(1988), pp. 381-408.

[Su] H. J. Sussmann, Rigorous results on the cubic sensor problem stochastic systems, in: The Mathematics of Filtering and Identification and Applications, M. Hazewinkel and J.C. Willems, (Eds.), Reidel, Dordrecht, The Netherlands, (1981), 637-648.

[Wu-Ya] X. WU AND S. S.-T. YAU, Classification of estimation algebras with state dimension 2, SIAM J. Control and Optimization, 44:3(2006), pp. 1039-1073.

[Ya] S. S.-T. YAU, Finite dimensional filters with nonlinear drift I: A class of filters including both Kalman-Bucy filters and Benes filters, Journal of Mathematic Systems, Estimation and Control, 4(1994), pp. 181-203.

[Ya-Hu1] S. S.-T. YAU AND G.-Q. Hu, Classification of finite dimensional estimation algebras of maximal rank with arbitrary state-space dimension and Mitter Conjecture, International Journal of Control, 78:10(2005), pp. 689-705.

[Ya-Hu2] S. S.-T. YAU AND G.-Q. Hu, Finite dimensional filters with nonlinear drift X: Explicit solution of DMZ equation, IEEE Transactions Automatic Control, 46:1(2001), pp. 142-148.

[Ya-Ra] S. S.-T. YAU AND A. RASOUlian, Classification of 4-dimensional estimation algebras, IEEE 
Transactions on Automatic Control, 44:4(1999), pp. 2312-2318.

[Y-W-W] S. S.-T. YAU, X. WU, AND W. S. WONG, Hessian matrix non-decomposition theorem, Mathematical Research Letter, 6(1999), pp. 1-11.

[Ya-Ya1] S.-T. YAU AND S. S.-T. YAU, Existence and uniqueness and decay estimates for the time dependent parabolic equation with application to Duncan-Mortensen-Zakai equation, Asian Journal of Mathematics, 2(1998), pp. 1079-1149.

[Ya-Ya2] S.-T. YAU AND S. S.-T. YAU, Real time solution of nonlinear filtering problem without memory I, Mathematical Research Letter, 7(2000), pp. 671-693.

[Ya-Ya3] S.-T. YAU AND S. S.-T. YAU, Real time solution of nonlinear filtering problem without memory II, preprint. 